\title{
Back Pain
}

National Institute of Neurological Disorders and Stroke (NINDS)

\section{Source}

National Institute of Neurological Disorders and Stroke (NINDS). Back Pain Information

Page.

Acute or short-term low back pain generally lasts from a few days to a few weeks. Most acute back pain is the result of trauma to the lower back or a disorder such as arthritis.

Pain from trauma may be caused by a sports injury, work around the house or in the garden, or a sudden jolt such as a car accident or other stress on spinal bones and tissues. Symptoms may range from muscle ache to shooting or stabbing pain, limited flexibility and range of motion, or an inability to stand straight. Chronic back pain is pain that persists for more than 3 months. It is often progressive and the cause can be difficult to determine. 